\title{
Understanding the expression of Toll-like receptors in Asian Indians predisposed to coronary artery disease
}

Arindam Maitra ${ }^{1}$, Jayashree Shanker ${ }^{1}$, Debabrata Dash ${ }^{1}$, Prathima Arvind ${ }^{1}$, Vijay V. Kakkar ${ }^{1,2}$

1Thrombosis Research Institute, India

2Thrombosis Research Institute, London, United Kingdom

Submitted: 5 May 2010

Accepted: 12 July 2010

Arch Med Sci 2011; 7, 5: 781-787

DOI: 10.5114/aoms.2011.25552

Copyright @ 2011 Termedia \& Banach

\section{Abstract}

Introduction: Toll-like receptors (TLRs) are an important link between innate and adaptive immunity.

Material and methods: Expression of TLR-2,TLR-4, and TLR-9 genes was assessed in 60 coronary artery disease (CAD) patients and 79 controls by SYBR Green 1 based real time PCR assay.

Results: Expression of the TLR-2 gene was found to be significantly elevated in cases $(1.295 \pm 0.09)$ compared to the controls $(1.033 \pm 0.08)(p=0.015)$ whereas expression of the $T L R-9$ gene was significantly lower in cases $(1.522 \pm 0.18)$ than in the controls $(2.165 \pm 0.16)(p=0.032)$. There was no difference in TLR-4 expression levels $(p=0.174)$. A significant correlation of $T L R-2$ was observed with TLR-4 $(r=0.803, p<0.0001)$ and TLR-9 $(r=0.264, p=0.003)$ as well as between TLR-4 and TLR-9 $(r=0.303, p=0.001)$. A significant association was seen between TLR 2 (OR 3.94, 95\% Cl 1.73-8.99, $p=0.001$ ) and TLR-9 (OR 0.297, $95 \% \mathrm{Cl} 0.131-0.672, p=0.004)$ with CAD after adjustment for age and gender. Statins did not affect $T L R$ gene expression.

Conclusions: The TLR-2,TLR-4 and TLR-9 genes exhibit a differential pattern of expression between CAD patients and controls in this Asian Indian cohort. This observation warrants further investigation, keeping in mind the infectious and inflammatory elements in perspective, in order to understand the true implications of TLR in the aetiopathology of CAD and consequent therapeutic implications.

Key words: Toll-like receptors, gene expression, coronary artery disease, Asian Indians.

\section{Introduction}

Atherosclerosis is considered to be an immunomodulatory disease, wherein infections by common pathogens and the ensuing inflammatory and immune response mechanisms play a significant role in the regulation of disease progression [1, 2]. Toll-like receptors (TLRs) are a family of pattern recognition receptors that serve as the first line of defence against invading microbes and/or tissue injury [3]. Endothelial cells, macrophages, adventitious fibroblasts and dendritic cells express TLRs in the atherosclerotic vessel wall. Twelve different families of TLRs have been discovered to date, of which TLR-1, TLR-2, TLR-4 and TLR-9 are key receptors implicated in the development and progression of coronary artery disease (CAD) $[4,5]$. The TLRs recognize a variety of bacterial and fungal

\author{
Corresponding author: \\ Dr. Jayashree Shanker \\ Thrombosis Research Institute \\ 258/A, Bommasandra \\ Industrial Area \\ Anekal Taluk \\ Bangalore 560099, India \\ Phone: 91-80-27835303, \\ 91-80-27835302 \\ E-mail: \\ jayashreeshanker@triindia.org.in
}


components that serve as their ligands peptidoglycans for TLR-2, lipopolysaccharides for TLR-4, flagellin for TLR-5 and unmethylated CpG motifs present in bacterial DNA for TLR-9 [6]. The TLRs initiate a cross talk between innate and adaptive immunity [7], resulting in activation of the nuclear factor- $\mathrm{KB}(\mathrm{NF} \kappa \mathrm{B})$ mediated inflammatory response via the direct regulation of immune responsive genes [8]. The TLR-mediated inflammation thus acts as an important pathogenic link between innate immunity and CAD [9].

Gene expression studies on the TLR family have shown that $T L R-1, T L R-2$ and $T L R-4$ genes are particularly elevated in human atherosclerotic lesions accompanied by parallel activation of TLRexpressing cells, as shown by the nuclear translocation of NFKB [10]. Recent findings have linked the TLR-mediated signalling cascade to established pathological determinants of plaque development and destabilization [11, 12]. Direct in vivo evidence on the pattern of TLR signalling in LDL receptor and apoE knock-out mice has shown that regulation of TLR expression is critical for maintaining immunological homeostasis [13]. TLRs have sparked great interest in recent times due to their potential for therapeutic manipulation of the innate immune system by way of alternate activation and down-regulation. TLRs can either serve as adjuvants for an immune-based vaccine by the induction of atheroprotective immunity [14] or as antagonists to TLR ligands, thereby arresting disease progression [15-17].

The present investigation is a part of the Indian Atherosclerosis Research Study (IARS), a large genetic epidemiological study on Asian Indians, where the underpinning objectives are to identify a potential vaccine to fight heart disease and to identify a strategic 'high risk' population for testing the vaccine. Given the key ability of TLRs to participate both in atheroprotective and proatherogenic immune responses, the objective of the present study was to assess the role of TLR in a cohort selected from the IARS by studying the expression levels of TLR-2, TLR-4 and TLR-9 as a pilot exploratory study.

\section{Material and methods}

All participants included in the study were selected from the IARS, an ongoing study, initiated and conducted by the Thrombosis Research Institute (TRI), India, to elucidate the involvement of genetic and non-genetic factors contributing to the aetiopathology of CAD among Asian Indians. The IARS participants were enrolled, based on predefined inclusion/exclusion criteria, from cardiac specialty hospitals in Bangalore and Mumbai situated in the southern and western regions of India. The CAD patients $(n=60)$ were selected from among the IARS probands who had a strong family history of CVD and age at onset of CAD $\leq 60$ years for men and $\leq 65$ years for women. All patients had angiographically confirmed presence of CAD. Patients having a history of primary myocardial disease or congenital heart disease were excluded from the study. The asymptomatic controls $(n=79)$ were matched for age and gender from among volunteers from the general population. The controls were considered healthy based on their ECG result and did not have a personal or family history of CAD. Individuals were considered to be diabetic or hypertensive based on self report of prescription medication. None of the patients were on insulin treatment. Individuals with concomitant infection were not enrolled in the study. Participation was by informed voluntary written consent. The IARS protocol was approved by the institutional ethics committee and is based on the Indian Council of Medical Research (ICMR) guidelines on Bioethics and the Helsinki Declaration for conducting research on human subjects [18].

\section{RNA isolation}

Around $22 \mathrm{ml}$ of blood sample was collected from all study participants. The serum, EDTA plasma and citrate plasma were separated into aliquots and preserved for biomarker assays while the blood pellet was used for DNA extraction. Around $3 \mathrm{ml}$ of EDTA whole blood was used for gene expression studies. Total RNA was extracted from fresh whole blood using the QIAamp Blood Mini Kit (Qiagen Inc., Valencia, CA, USA) for RNA extraction as per the recommended protocol. Purified RNA was converted to cDNA using the cDNA archive kit (Applied Biosystems Inc., Foster Coty, CA, USA) and stored at $-80^{\circ} \mathrm{C}$.

\section{TLR assays and data analysis}

SYBR Green I based cDNA specific real time PCR assays for $T L R-2, T L R-4, T L R-9$ and GAPDH genes were designed and developed in house. Table I shows the forward and reverse primers as well as the PCR conditions used for the SYBR green assay. Expression analysis of $T L R-2, T L R-4$ and $T L R-9$ genes was performed with replicates of the CDNA, using the GAPDH gene as an endogenous control. Relative quantitation of gene expression was performed by a method described previously [19]. The intra-assay coefficient of variation (CV) of the relative quantities obtained for TLR-2, TLR-4 and TLR-9 was 5.9\%, 6.4\% and $7.6 \%$, respectively, while the inter-assay CV for the same was estimated to be $7.3 \%, 9.4 \%$ and $8.1 \%$, respectively.

\section{Statistical analysis}

Multivariate analysis of fold changes in relative gene expression was performed with and without 
Table I. Primer sequences used for TLR genes and GAPDH2 with PCR conditions

\begin{tabular}{|c|c|c|c|}
\hline Genes & Primers & Sequence & PCR condition \\
\hline \multirow[t]{2}{*}{$T L R 2$} & $1642 \mathrm{~F}$ & CCAGGCTCTGGTGCTGACAT & \multirow{8}{*}{$\begin{array}{l}\text { Initial denaturation }-95^{\circ} \mathrm{C} \text { for } 10^{\prime} \text {, } \\
40 \text { cycles of denaturation at } 95^{\circ} \mathrm{C} \text { for } 15 \mathrm{~s} \text {, } \\
\text { annealing/extension at } 60^{\circ} \mathrm{C} \text { for } 1^{\prime} \text {, } \\
\text { final dissociation step }\end{array}$} \\
\hline & $1713 R$ & AGACTGCCCAGGGAAGAAAAA & \\
\hline \multirow[t]{2}{*}{ TLR4 } & $1951 \mathrm{~F}$ & GGCATGCCTGTGCTGAGTT & \\
\hline & $2020 R$ & TGAGGACCGACACACCAATGA & \\
\hline \multirow[t]{2}{*}{ TLR9 } & $1533 \mathrm{~F}$ & AGTCAATGGCTCCCAGTTCCT & \\
\hline & $1626 R$ & CGTGAATGAGTGCTCGTGGTA & \\
\hline \multirow[t]{2}{*}{ GAPDH2 } & $3210 \mathrm{~F}$ & CAAGGCTGTGGGCAAGGT & \\
\hline & $3268 \mathrm{R}$ & GGAAGGCCATGCCAGTGA & \\
\hline
\end{tabular}

adjustment for traditional risk factors, namely, age, gender, history of smoking, hypertension and diabetes. The correlation amongst the expression levels of $T L R-2, T L R-4$ and $T L R-9$ genes was assessed by multivariate statistics and Pearson's correlation test. Logistic regression analysis was performed to assess the association of TLRs with CAD after eliminating the effect of age and gender. SPSS version 15 was employed for statistical analysis and a nominal $p$ value of 0.05 or less was considered as statistically significant.

\section{Results}

The clinical characteristics of the study participants are provided in Table II. There was no significant difference between cases and controls with respect to the mean age, gender, systolic and diastolic blood pressure, body mass index, waisthip-ratio, and smoking. Frequency of diabetes and hypertension was, however, significantly higher in the cases than in the controls $(p<0.001)$. Of the 60 CAD patients, 21 of them had suffered a myocardial infarction (MI) event, while 30 subjects had stable angina disease. Clinical details were missing for 9 individuals who had proven CAD based on a cardiologist's diagnosis. The mean age at onset of CAD was $50 \pm 7.4$ years for males and $56.7 \pm 4.2$ years for females.

\section{Gene expression analysis}

The expression levels of the TLR-2 gene was found to be significantly elevated in the cases (1.295 $\pm 0.09)$ as compared to the controls (1.033 \pm 0.08$)$ $(p=0.015)$. On the other hand, the expression levels for the TLR-9 gene was significantly lower in the cases $(1.522 \pm 0.18)$ than the controls $(2.165 \pm 0.16)$ $(p=0.032)$ while there was no significant differences in the expression levels for the TLR-4 gene $(p=0.174)$ (Figure 1$)$. This pattern did not change following covariate adjustment for age, gender, history of smoking, hypertension or diabetes (Figure 2).

We observed a significant correlation of TLR-2 with TLR-4 $(r=0.803, p<0.0001)$ and TLR-9 $(r=0.264, p=0.003)$ as well as between TLR-4 and TLR-9 $(r=0.303, p=0.001)$, respectively, after

Table II. Clinical characteristics of study participants

\begin{tabular}{|lccc|}
\hline Parameter & Cases $(n=60)$ & Controls $(n=79)$ & Value of $p$ \\
\hline Age [years] & $52.19 \pm 7.39$ & $51.76 \pm 8.27$ & 0.935 \\
\hline Gender $(\mathrm{M} / \mathrm{F})$ & $53 / 7$ & $72 / 7$ & 0.586 \\
\hline Body mass index $\left[\mathrm{kg} / \mathrm{m}^{2}\right]$ & $25.6 \pm 0.49$ & $24.97 \pm 0.48$ & 0.369 \\
\hline Waist/hip ratio & $0.97 \pm 0.01$ & $0.96 \pm 0.01$ & 0.302 \\
\hline Systolic blood pressure $[\mathrm{mmHg}]$ & $121.34 \pm 1.77$ & $127.03 \pm 2.21$ & 0.06 \\
\hline Diastolic blood pressure $[\mathrm{mmHg}]$ & $77.93 \pm 1.12$ & $81.0 \pm 1.17$ & 0.069 \\
\hline Height [cm] & $166.12 \pm 1.11$ & $165.52 \pm 0.83$ & 0.66 \\
\hline Weight [kg] & $70.44 \pm 1.2$ & $68.28 \pm 1.4$ & 0.265 \\
\hline Diabetes [\%] & $27(47.4)$ & $5(6.4)$ & $<0.0001$ \\
\hline Hypertension [\%] & $31(55.4)$ & $6(7.7)$ & $<0.0001$ \\
\hline Statin [\%] & $30(50.0)$ & 0 & - \\
\hline Smoking (ever) & $27(46.6)$ & $31(39.7)$ & 0.427 \\
\hline
\end{tabular}




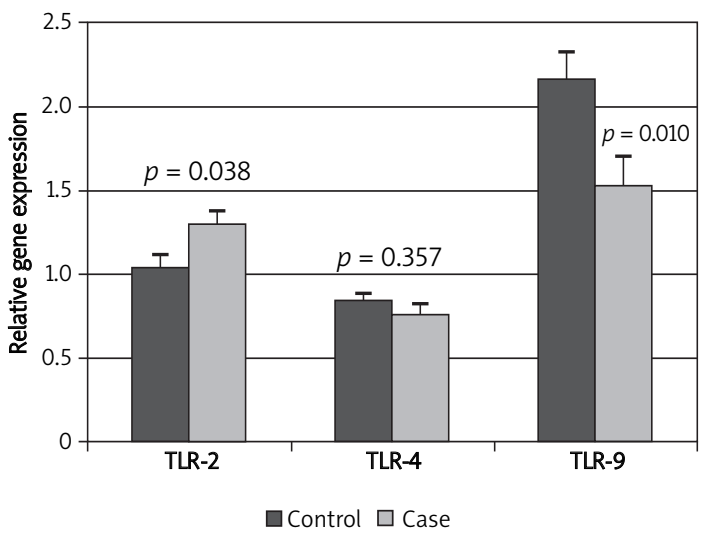

Figure 1. Relative gene expression in $T L R-2, T L R-4$ and $T L R-9$ between cases and controls before adjusting for conventional risk factors

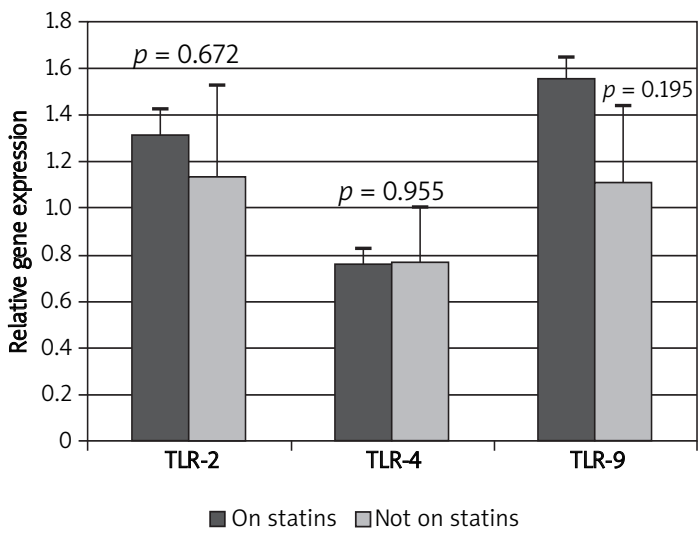

Figure 3. Effect of statin usage on fold changes in $T L R$ gene expression in CAD patients

adjusting for age, gender, diabetes, hypertension and smoking. There was a significant association of TLR-2 (OR 3.94, 95\% Cl 1.73-8.99, $p=0.001$ ) and TLR-9 (OR 0.297, 95\% Cl 0.131-0.672, $p=0.004$ ) with $C A D$, after adjustment for age and gender. The predictive probability of the TLRs to accurately classify the CAD cases and controls based on the ROC analysis (AUC value) was $0.69(p<0.000)$ (Figure 3) with overall classification of $62.6 \%$ of the cohort.

A comparison of the $T L R$ gene expression profile between the study participants on statin therapy and those who were not on the lipid-lowering drug revealed that there was no significant difference in the relative expression profile for all the three $T L R$ genes (Figure 3).

\section{Discussion}

Given the high global prevalence of CVD and its co-morbidities, there is an urgent need to identify novel ways of predicting and managing cardiovascular risk. It is common knowledge that

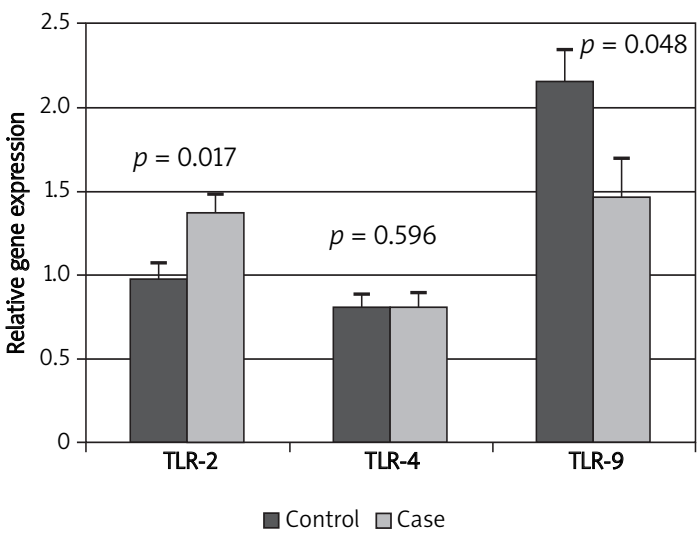

Figure 2. Relative gene expression in TLR-2, TLR-4 and TLR-9 between cases and controls after adjusting for age, gender, hypertension, diabetes and smoking

conventional risk factors account for only $50 \%$ of the disease prevalence and existing modalities for addressing this risk are primarily through life style interventions and/or through use of the limited choice of medications available in the market today. Based on the clinico-epidemiological and experimental studies, there is a growing understanding that infection may play a significant role in the initiation and progression of atherosclerotic disease [20-22]. Therefore, novel therapies such as modulation of the immune system have opened up unique opportunities to redress the growing concern of CVD burden. In this connection, the TLRS play a critical role in bridging the gap between immune response and cardiovascular disease progression. Lessons learnt from knock out mouse models (apoE -/-, double knock outs such as apoE -/- and TLR-4 -/- or apoE -/- and CD14 -/-) have provided strong mechanistic evidence that TLRs are responsible for the exacerbation of atherosclerotic disease [23]. Mechanisms of TLR action are manifold - ability to directly interfere with cholesterol metabolism in the macrophages by serving as a ligand for minimally modified LDL, a proatherogenic and proinflammatory lipoprotein [24], interaction on the endothelial cell surface resulting in the enhanced secretion of IL-18, a potent proinflammatory cytokine [25], and cross reaction between bacterial and human heat shock protein 60 (HSP60) through molecular mimicry. The signalling here is mediated through TLR-2 and/or TLR-4, leading to the activation of NFKB dependent proinflammatory gene targets [26-28] and consequent accumulation of lipids and leukocytes in the atheroma [3]. Pathological changes that occur during the atherosclerotic disease process release endogenous inflammatory molecules and oxidatively modified lipids that serve as ligands that engage the TLRs and activate their expression [29]. 
The Bruneck study provided the first epidemiological evidence that circulating pro-inflammatory mediators such as bacterial lipopolysaccharides (LPS) can constitute a strong risk factor for atherosclerosis development [30]. Subsequently, TLR-1, TLR-2 and TLR-4 were shown to be markedly elevated in human atherosclerotic plaque specimens [10] and in the medium and large arterial trees [31].

The above studies highlight the prominent role of TLRs as an emerging biomarker of atherosclerosis and a potential therapeutic target against CAD. Given this topical interest in TLRs, the present study was an exploratory study to understand the role of circulating TLR expression in CAD patients with strong familial predisposition to CAD. Our initial findings reveal that there is a differential pattern of gene expression in CAD patients as compared to the controls. In our study, the expression of TLR-2, was found to be significantly elevated while $T L R-9$ was lower in CAD patients as compared to controls. On the other hand, there was no difference in the levels of $T L R-4$ expression between the two groups. High levels of TLR-2 expression were reported on the surface of circulating monocytes, independent of established risk factors [32]. Genetic variations in the TLRs can attenuate (Asp299Gly in TLR-4) [33] or enhance the inflammatory and immune response to pathogenic challenge and thereby influence the pathogenesis or outcome of disease [34, 35]. Novel mutations have been identified in TLR-1 (Ser150Gly and Val220Met) and TLR-2 (Phe670Leu) that alter the ability of TLRs to recognize specific PAMPs associated with infection by Mycobacterium avium subsp. paratuberculosis (MAP) [36].

In the IARS, we have measured the antibody titres to various common pathogens such as Cytomegalovirus, Chlamydia pneumoniae, Helicobacter pylori and Herpes simplex virus 1. Seropositivity to the first three pathogens was found to be significantly higher in CAD patients $(p<0.05)$ as compared to their unaffected family members; also, the number of CAD patients was higher in the group with multiple rather than single infections (data unpublished), supporting the hypothesis that total pathogen burden rather than single infections are an important aetiopathological factor for $C A D$ $[37,38]$. Given this background knowledge, it is not surprising that we observed enhanced expression for TLR-2 in our patients selected from the IARS cohort. However, these preliminary findings have to be extended to a larger cohort to understand the true implications of the fold changes amongst the various TLRs and their downstream modulation of proinflammatory cytokines. In a recent report on the analysis of vessel-specific profiling of various TLRs in the medium and large arteries, it has been shown that while TLR-2 and TLR-4 are ubiquitously expressed, TLR-7 and TLR-9 may be infrequent and the other TLRs may show selective expression [31]. This again has to be further validated in the present context. In a recent study, a decrease in the expression of TLR-4 was shown to be associated with progression of cervical neoplasia in cases of infections with human papillomavirus (HPV) [39].

Yet another interesting study on Sprague-Dawley rats has shown that the underlying mechanism of ischemic preconditioning during repeated ischemic reperfusion might be related to decreased levels of TLR-4 expression and reduced levels of proinflammatory cytokines, TNF- $\alpha$ and IL-1 $\beta$ [40].

TLR signalling has been shown to increase plaque vulnerability through myeloid differentiation primary response gene 88 (MyD88), a signalling adaptor shared by most $T L R$ genes $[17,40]$. In our study over $41 \%$ of CAD patients had experienced an $\mathrm{Ml}$ event, suggesting the possible association of enhanced TLR expression with adverse cardiovascular outcome.

Statin treatment, primarily advocated for lowering of cholesterol levels, has been shown to decrease TLR-4 signalling through attenuation of the LPS responsiveness [41]. In contrast, such signalling has also been shown to enhance TLRmediated cytokine expression in astrocytes through negative feedback regulation of the Rho proteins [42]. However, in the present study, statins did not appear to influence the pattern of TLR expression in CAD patients.

Although our pilot study reveals alterations in expression levels of $T L R-2$ and $T L R-9$ genes in the present study, there are certain limitations such as the small sample size, increased prevalence of comorbidities such as diabetes and hypertension and use of statins in the patient group as compared to the controls. These are being addressed in a further study involving well-matched control groups. Furthermore, we need to evaluate the influence of altered TLR expression on downstream cytokines that might provide stronger evidence on the mode of TLR regulation in atherosclerosis. An interesting association has been reported on the enhanced expression of IL-10-dependent elevated expression of TLR-9 by Treg cells through induction by CD3+ $T$ cells in the presence of vitamin $D$, which has significant implications for vaccine design [43]. Developing a vaccine against atherosclerosis is one of our primary objectives, and the report by Urry et al. provides critical insights into mechanism of TLR regulation and the balance achieved by the $T$ regulatory (Treg) cells in coordinating an effective immune response. We have observed that over $97 \%$ of the CAD patients have chronic vitamin D deficiency in a pilot study in the IARS (Insert reference \# 44 - PMID: 216110492). The reported association of vitamin D mediated immune response of TLRS opens up exciting avenues for detailed investigations. 
In conclusion, the present study provides early leads on the differential pattern in the expression levels of $T L R-2, T L R-4$ and $T L R-9$ genes in CAD patients with strong familial predisposition to CVD. This is in line with the emerging paradigm that atherosclerosis is a pathogen-mediated immunomodulatory disease. Further work is required to understand the true profile of TLRs, their correlation with presence of infection and other inflammatory biomarkers, as well as their functional relevance to the pathology of coronary artery disease in Asian Indians.

\section{Acknowledgments}

We thank the Trustees of TRI India and London for their support and encouragement. We are very grateful to the Tata Social Welfare Trust, the Department of Biotechnology, Garfield Weston Foundation and the Elizabeth \& Emmanuel Kaye Foundation for the financial support extended to the Indian Atherosclerosis Research Study and the Thrombosis Research Institute India. We thank the doctors at Narayana Hrudayalaya and other hospitals in Bangalore and the Asian Heart Institute and Research Center in Mumbai for providing clinical assistance. We appreciate the efforts of the clinical staff, the data entry team and the administrative members of TRI India and London. We profusely thank the study participants for their voluntary participation in our study.

\section{References}

1. Hansson GK. Inflammation, atherosclerosis, and coronary artery disease. N Engl J Med 2005; 352: 1685-95.

2. Nilsson J, Hansson GK, Shah PK. Immunomodulation of atherosclerosis: implications for vaccine development. Arterioscler Thromb Vasc Biol 2005; 25: 18-28.

3. Curtiss LK, Tobias PS. Emerging role of Toll-like receptors in atherosclerosis. J Lipid Res 2009; 50 Suppl: S340-5.

4. Akira S, Takeda K, Kaisho T. Toll-like receptors: critical proteins linking innate and acquired immunity. Nat Immunol 2001; 2: 675-80.

5. Takeda K, Kaisho T, Akira S. Toll-like receptors. Annu Rev Immunol 2003; 21: 335-76.

6. Brikos C, O'Neill LA. Signalling of toll-like receptors. Handb Exp Pharmacol 2008; 183: 21-50.

7. Oude Nijhuis MM, van Keulen JK, Pasterkamp G, Quax $\mathrm{PH}$, de Kleijn DP. Activation of the innate immune system in atherosclerotic disease. Curr Pharm Des 2007; 13 . 983-94.

8. Frantz S, Ertl G, Bauersachs J. Mechanisms of disease: Toll-like receptors in cardiovascular disease. Nat Clin Pract Cardiovasc Med 2007; 4: 444-54.

9. Tobias PS, Curtiss LK. Toll-like receptors in atherosclerosis. Biochem Soc Trans 2007; 35: 1453-5.

10. Edfeldt K, Swedenborg J, Hansson GK, Yan ZQ. Expression of toll-like receptors in human atherosclerotic lesions: a possible pathway for plaque activation. Circulation 2002; 105: 1158-61.
11. Ishikawa $Y$, Satoh $M$, Itoh T, et al. Local expression of Tolllike receptor 4 at the site of ruptured plaques in patients with acute myocardial infarction. Clin Sci (Lond) 2008; 115: 133-40.

12. Satoh M, Ishikawa Y, Minami Y, Takahashi Y, Nakamura M. Role of Toll like receptor signaling pathway in ischemic coronary artery disease. Front Biosci 2008; 13: 6708-15.

13. Takeda K, Akira S. TLR signaling pathways. Semin Immunol 2004; 16: 3-9.

14. Hansson GK, Nilsson J. Vaccination against atherosclerosis? Induction of atheroprotective immunity. Semin Immunopathol 2009; 31: 95-101.

15. Bhattacharjee RN, Akira S. Modifying toll-like receptor 9 signaling for therapeutic use. Mini Rev Med Chem 2006; 6: 287-91.

16. Kanzler H, Barrat FJ, Hessel EM, Coffman RL. Therapeutic targeting of innate immunity with Toll-like receptor agonists and antagonists. Nat Med 2007; 13: 552-9.

17. Monaco C, Gregan SM, Navin TJ, et al. Toll-like receptor-2 mediates inflammation and matrix degradation in human atherosclerosis. Circulation 2009; 120: 2462-9.

18. Kumar NK. Bioethics activities in India. East Mediterr Health J 2006; 12 Suppl 1: S56-65.

19. Pfaffl MW. A new mathematical model for relative quantification in real-time RT-PCR. Nucleic Acids Res 2001; 29: e45.

20. Epstein SE. The multiple mechanisms by which infection may contribute to atherosclerosis development and course. Circ Res 2002; 90: 2-4.

21. Epstein SE, Zhou YF, Zhu J. Infection and atherosclerosis: emerging mechanistic paradigms. Circulation 1999; 100: e20-8.

22. Deniset JF, Cheung PK, Dibrov E, et al. Chlamydophila pneumoniae infection leads to smooth muscle cell proliferation and thickening in the coronary artery without contributions from a host immune response. Am J Pathol 2010; 176: 1028-37.

23. Michelsen KS, Doherty TM, Shah PK, Arditi M. TLR signaling: an emerging bridge from innate immunity to atherogenesis. J Immunol 2004; 173: 5901-7.

24. Miller YI, Viriyakosol S, Worrall DS, et al. Toll-like receptor 4-dependent and -independent cytokine secretion induced by minimally oxidized low-density lipoprotein in macrophages. Arterioscler Thromb Vasc Biol 2005; 25: 1213-9.

25. Walton KA, Cole AL, Yeh $M$, et al. Specific phospholipid oxidation products inhibit ligand activation of toll-like receptors 4 and 2. Arterioscler Thromb Vasc Biol 2003; 23: 1197-203.

26. Bulut Y, Faure E, Thomas L, et al. Chlamydial heat shock protein 60 activates macrophages and endothelial cells through Toll-like receptor 4 and MD2 in a MyD88dependent pathway. J Immunol 2002; 168: 1435-40.

27. Vabulas RM, Ahmad-Nejad P, da Costa C, et al. Endocytosed HSP60s use toll-like receptor 2 (TLR2) and TLR4 to activate the toll/interleukin-1 receptor signaling pathway in innate immune cells. J Biol Chem 2001; 276: 31332-9.

28. Xu Q. Infections, heat shock proteins, and atherosclerosis. Curr Opin Cardiol 2003; 18: 245-52.

29. Ionita MG, Arslan F, de Kleijn DP, Pasterkamp G. Endogenous inflammatory molecules engage Toll-like receptors in cardiovascular disease. J Innate Immun 2010; 2: $307-15$. 
30. Wiedermann CJ, Kiechl S, Dunzendorfer S, et al. Association of endotoxemia with carotid atherosclerosis and cardiovascular disease: prospective results from the Bruneck Study. J Am Coll Cardiol 1999; 34: 1975-81.

31. Pryshchep O, Ma-Krupa W, Younge BR, Goronzy JJ, Weyand CM. Vessel-specific Toll-like receptor profiles in human medium and large arteries. Circulation 2008; 118: 1276-84.

32. Kuwahata S, Fujita S, Orihara K, et al. High expression level of Toll-like receptor 2 on monocytes is an important risk factor for arteriosclerotic disease. Atherosclerosis 2010; 209: 248-54.

33. Kiechl S, Lorenz E, Reindl M, et al. Toll-like receptor 4 polymorphisms and atherogenesis. N Engl J Med 2002; 347: 185-92.

34. Balistreri CR, Candore G, Listi F, et al. Role of TLR4 polymorphisms in inflammatory responses: implications for unsuccessful aging. Ann N Y Acad Sci 2007; 1119: 203-7.

35. Corr SC, O'Neill LA. Genetic variation in Toll-like receptor signalling and the risk of inflammatory and immune diseases. J Innate Immun 2009; 1: 350-7.

36. Bhide MR, Mucha R, Mikula I Jr, et al. Novel mutations in TLR genes cause hyporesponsiveness to Mycobacterium avium subsp. paratuberculosis infection. BMC Genet 2009; 10: 21.

37. Epstein SE, Zhu J, Burnett MS, et al. Infection and atherosclerosis: potential roles of pathogen burden and molecular mimicry. Arterioscler Thromb Vasc Biol 2000; 20: $1417-20$

38. Zhu J, Quyyumi AA, Norman JE, et al. Effects of total pathogen burden on coronary artery disease risk and C-reactive protein levels. Am J Cardiol 2000; 85: 140-6.

39. Yu L, Wang L, Li M, et al. Expression of toll-like receptor 4 is down-regulated during progression of cervical neoplasia. Cancer Immunol Immunother 2010; 59: 1021-8.

40. Yu M, Wen N, Wenzhong Z, Yuanchang X, Xiaoming D, Yongjin $\mathrm{L}$. Effect of repeated ischaemic preconditioning on TLR4 and proinflammatory cytokines TNF-alpha and IL-1beta in myocardial ischaemia-reperfusion injury in a rat model. Arch Med Sci 2010; 6: 843-7.

41. Methe H, Kim JO, Kofler S, Nabauer M, Weis M. Statins decrease Toll-like receptor 4 expression and downstream signaling in human CD14+ monocytes. Arterioscler Thromb Vasc Biol 2005; 25: 1439-45.

42. Krasowska-Zoladek A, Banaszewska M, Kraszpulski M, Konat GW. Kinetics of inflammatory response of astrocytes induced by TLR 3 and TLR4 ligation. J Neurosci Res 2007; 85: 205-12.

43. Urry Z, Xystrakis E, Richards DF, et al. Ligation of TLR9 induced on human IL-10-secreting Tregs by 1alpha, 25-dihydroxyvitamin D3 abrogates regulatory function. J Clin Invest 2009; 119: 387-98. 